\title{
Inefficient volatile loss from the Moon-forming disk: reconciling the giant impact hypothesis and a wet Moon
}

\author{
Miki Nakajima $^{1,2}$, David J. Stevenson ${ }^{2}$ \\ ${ }^{1}$ Department of Terrestrial Magnetism, Carnegie Institution for Science, 5241 Broad Branch Rd NW, Washington, DC 20015, USA. \\ ${ }^{2}$ Division of Geological and Planetary Sciences, California Institute of Technology, 1200 E California Blvd, MC 150-21, Pasadena, CA 91125, \\ USA.
}

\begin{abstract}
The Earth's Moon is thought to have formed from a circumterrestrial disk generated by a giant impact between the proto-Earth and an impactor approximately 4.5 billion years ago. Since this impact was energetic, the disk would have been hot $(4000-6000 \mathrm{~K})$ and partially vaporized $(20-100 \%$ by mass). This formation process is thought to be responsible for the geochemical observation that the Moon is depleted in volatiles (e.g., $\mathrm{K}$ and Na). To explain this volatile depletion, some studies suggest the Moon-forming disk was rich in hydrogen, which was dissociated from water, and it escaped from the disk as a hydrodynamic wind accompanying heavier volatiles (hydrodynamic escape). This model predicts that the Moon should be significantly depleted in water, but this appears to contradict some of the recently measured lunar water abundances and $\mathrm{D} / \mathrm{H}$ ratios that suggest that the Moon is more water-rich than previously thought. Alternatively, the Moon could have retained its water if the upper parts (low pressure regions) of the disk were dominated by heavier species because hydrogen would have had to diffuse out from the heavy-element rich disk, and therefore the escape rate would have been limited by this slow diffusion process (diffusion-limited escape). To identify which escape the disk would have experienced and to quantify volatiles loss from the disk, we compute the thermal structure of the Moon-forming disk considering various bulk water abundances (100-1000 ppm) and mid-plane disk temperatures $(2500-4000 \mathrm{~K})$. Assuming that the disk consists of silicate $\left(\mathrm{SiO}_{2}\right.$ or $\left.\mathrm{Mg}_{2} \mathrm{SiO}_{4}\right)$ and water and that the disk is in the chemical equilibrium, our calculations show that the upper parts of the Moon-forming disk are dominated by heavy atoms or molecules ( $\mathrm{SiO}$ and $\mathrm{O}$ at $T_{\text {mid }}>2500-2800 \mathrm{~K}$ and $\mathrm{H}_{2} \mathrm{O}$ at $T_{\text {mid }}<2500-2800$ $\mathrm{K}$ ) and hydrogen is a minor species. This indicates that hydrogen escape would have been diffusion-limited, and therefore the amount of lost water and hydrogen would have been small compared to the initial abundance assumed. This result indicates that the giant impact hypothesis can be consistent with the water-rich Moon. Furthermore, since the hydrogen wind would have been weak, the other volatiles would not have escaped either. Thus, the observed volatile depletion of the Moon requires another mechanism.
\end{abstract}

Keywords: Moon; giant impact; volatiles; lunar water; volatile loss; hydrodynamic escape https://doi.org/10.1016/j.epsl.2018.01.026

\section{Introduction}

It is widely accepted that the Earth's Moon formed by a collision between the proto-Earth and an impactor approximately 4.5 billion years ago (Hartmann and Davis, 1975; Cameron and Ward, 1976). This impact created a partially vaporized disk around the planet, from which the Moon accreted. In the standard version of this hypothesis, the impactor was approximately Mars-sized

Email address: mnakaj ima@carnegiescience.edu (Miki Nakajima $^{1,2}$ )

Preprint submitted to Earth and Planetary Science Letters and the impact velocity was close to the escape velocity (Canup and Asphaug, 2001). This model has been favored because it can explain several observed aspects of the Earth-Moon system, such as its angular momentum, the lunar mass, and the small iron core of the Moon. However, the model cannot easily explain the observation that the Earth and Moon have identical or strikingly similar isotopic ratios (e.g., oxygen and tungsten, Wiechert et al. 2001; Herwartz et al. 2014; Young et al. 2016; Kruijer et al. 2015; Touboul et al. 2015) given that the impact simulations indicate that most of the disk 
materials originate from the impactor, which presumably had different isotopic ratios from the Earth. It may be, however, possible that the impactor happened to have similar isotopic ratios to those of Earth because the inner solar system may have been well-mixed (Dauphas, 2017). This idea might bolster the standard model, but it still requires an explanation for the identical tungsten isotopic ratios.

Alternatively, Ćuk and Stewart (2012) suggest that a small impactor hit a rapidly rotating Earth while Canup (2012) suggests that two half Earth-sized objects collided. In these models, the composition of the disk is similar to that of the Earth, and therefore, the isotopic similarities could be naturally explained. These new models are promising alternatives, but they may predict that the Earth's mantle becomes mixed by the energetic impact (Nakajima and Stevenson, 2015). This appears to contradict observed anomalies of short-lived isotopes indicating that the Earth has never been completely mixed (e.g., ${ }^{182} \mathrm{Hf}^{182} \mathrm{~W}$, Willbold et al. 2011; Touboul et al. 2012; Rizo et al. 2016; Mundl et al. 2017 and noble gases, Mukhopadhyay 2012). A recent suggestion that the Moon could have formed as the outcome of merging of smaller Moons and multiple impacts (Rufu et al., 2017) revives an old idea requiring some specific, perhaps unlikely dynamical conditions to be acceptable.

In addition to the isotopic ratios of the Earth-Moon system, the chemical compositions provide further essential information. The giant impact has been thought to be at least partly responsible for the observation that the Moon is depleted in volatiles, such as $\mathrm{K}, \mathrm{Rb}, \mathrm{Na}$, and other volatile elements (e.g., Krähenbühl et al., 1973; Ringwood et al., 1987). Smaller K/Th and K/U ratios of the Moon than those of the Earth also indicate that the Moon is depleted in volatiles ( $\mathrm{K}$ is more volatile than $\mathrm{Th}$ and U) (Tera et al., 1974). The Moon-forming disk was hot and partially vaporized (up to $4000-5000 \mathrm{~K}$ and 20$30 \%$ for the standard case and $6000-7000 \mathrm{~K}$ and $80-90 \%$ for the recent models, Nakajima and Stevenson 2014). Hydrogen, which would have been dissociated from water at this high temperature, may have escaped from the hot Moon-forming disk as a wind (hydrodynamic escape) together with heavier atoms and molecules (Genda and Abe, 2003; Desch and Taylor, 2013).

This model predicts that the Moon also lost a significant amount of water, but this appears to be inconsistent with some of the measured lunar water abundances. Determining the bulk lunar water abundance is an active area of research; based on these measurements and modeling of the lunar interior evolution (e.g., Boyce et al., 2010; McCubbin et al., 2010; Hauri et al.,
2011; Hui et al., 2013; Saal et al., 2013), the bulk water content of the Moon has been estimated to range from $<10 \mathrm{ppm}$ (Elkins-Tanton and Grove, 2011) to a few hundred ppm (Hui et al., 2013; Hauri et al., 2011, 2015; Milliken and Li, 2017). Lin et al. (2016) suggest that the crustal thickness estimated by GRAIL (34-43 $\mathrm{km}$, Wieczorek et al. 2013) can be consistent with an initially deep ( $700 \mathrm{~km})$ lunar magma ocean with presence of water (270 - $1650 \mathrm{ppm})$. On the other hand, work using $\mathrm{Cl}$ and $\mathrm{F}$ in addition to $\mathrm{H}$ in apatite suggests that the water content of the Moon can be much lower (e.g., Boyce et al., 2014). These results cover a wide range: the Moon could be "wet", which indicates here that the Moon is as water-rich as Earth (Earth's bulk water abundance is estimated as a couple of hundred ppm, McDonough and Sun 1995) or could be drier $(<100 \mathrm{ppm})$. Needless to say, the possibility that water is heterogeneously distributed within the Moon (Robinson and Taylor, 2014) makes it even more difficult to estimate the bulk lunar water abundance based on a small set of samples.

If a significant amount of water escaped from the disk, the lunar $\mathrm{D} / \mathrm{H}$ ratios should be more enhanced than that of the Earth because $\mathrm{H}$ is lighter and would have escaped more efficiently than D. However, analyses of pristine lunar water suggest that lunar $\mathrm{D} / \mathrm{H}$ ratios may be comparable to the terrestrial values, which may indicate that water loss was insignificant (e.g., Saal et al., 2013). It should be noted that measuring the bulk content of the indigenous water and $\mathrm{D} / \mathrm{H}$ ratio is a very challenging task because the available lunar samples are limited and because a number of processes, including fractional crystallization, degassing, solar wind irradiation, and cosmic-ray spallation, would likely alter the original values.

Thus, the Moon is depleted in some volatiles, but it may or may not be depleted in water. To understand the history of the lunar volatiles, we propose to reevaluate the water loss mechanism. Desch and Taylor (2013) suggest that hydrodynamic escape could have occurred and blew off the disk atmosphere when the disk temperature is $2000 \mathrm{~K}$ and the mean molecular weight of the disk is $\bar{m}=6 \mathrm{~g} \mathrm{~mol}^{-1}$ (i.e., water in the disk was dissociated to $2 \mathrm{H}$ and $\mathrm{O}$ ). Conventionally, hydrodynamic escape from a planetary atmosphere occurs when the Jeans parameter $\lambda \equiv G M_{\oplus} \bar{m} / R T r^{\prime}$ is smaller than $\sim 2$ (Parker, 1963) (the exact number of this criterion can vary depending on the atmospheric composition, Volkov et al. 2011, and the geometry, Desch and Taylor 2013). Here, $G$ is the gravitational constant, $M_{\oplus}$ is the Earth mass, $R$ is the gas constant, $T$ is the temperature, and $r^{\prime}$ is the distance from the planet. The work done 
by Desch and Taylor (2013) is certainly insightful, but an important aspect here is that this criterion of $\lambda$ has been developed for a gas that behaves as a material with a single molecular weight (for example the solar wind, which is primarily hydrogen), and it is necessary to understand if this model is applicable to the specific system of interest. If the disk were dominated by heavier elements that were gravitationally bound (i.e., the escape fluxes of the heavy elements were negligible), the hydrodynamic escape model is no longer valid. For hydrogen to escape from a disk dominated by heavier elements, it must diffuse out from the heavy elements that are gravitationally bound to the planet-disk system. Thus, the hydrogen escape rate is limited by this diffusion process and this is called diffusion-limited escape, which is much slower than the "blow off" hydrodynamic escape. This type of hydrogen escape likely occurred from early planetary atmospheres (e.g., Hunten, 1973; Zahnle et al., 1990).

As an example, consider a disk that is dominated by a light element $i$ and heavy element $j$. We assume here that $i$ and $j$ are hydrogen and oxygen, respectively, and that their mole fractions are $f_{i}=\frac{n_{i}}{\left(n_{i}+n_{j}\right)}=f_{\mathrm{H}}=0.1$, where $n_{i}$ and $n_{j}$ are the number densities of the element $i$ and $j$, respectively. The upper limit of the diffusionlimited escape rate is described as (Hunten, 1973),

$$
\phi_{l}=b_{i j} f_{i}\left(\frac{1}{H_{j}}-\frac{1}{H_{i}}\right) \sim b_{i j} f_{i} / H_{j},
$$

where $b_{i j}$ is the binary collision parameter between elements $i$ and $j, f_{i}$ is the mole fraction of the element $i, H_{i}\left(=R T / m_{i} g\right.$, where $m_{i}$ is the molecular weight of the element $i$ ) is the scale height of the element $i$. The subscripts $i$ and $j$ represent the elements $i$ and $j$, respectively. $R$ is the gas constant, and $g$ is the gravity. The last approximation is valid when $H_{i} \gg H_{j}$. Here, we are assuming that the heavy element $j$ is not escaping from the system.

Under the hard-sphere approximation, $b_{i j}$ is described as (Chamberlain and Hunten, 1987),

$$
b_{i j}=\frac{3}{64 Q}\left(2 \pi R T \frac{m_{i}+m_{j}}{m_{i} m_{j}}\right)^{\frac{1}{2}},
$$

where

$$
Q=\frac{\pi}{16}\left(\sigma_{i}+\sigma_{j}\right)^{2} .
$$

$\sigma$ is the collision diameter. Assuming $i$ is atomic hydrogen and $j$ is atomic oxygen, the hydrogen escape flux becomes $1.83 \times 10^{15}$ atoms $\mathrm{m}^{-2} \mathrm{~s}^{-1}\left(\sigma_{i}=2 \times 53 \mathrm{pm}, \sigma_{j}=\right.$ $2 \times 60 \mathrm{pm}, m_{i}=1 \mathrm{~g} \mathrm{~mol}^{-1}, m_{j}=16 \mathrm{~g} \mathrm{~mol}^{-1}, T=2000 \mathrm{~K}$, $f_{i}=0.1, r=3 R_{\oplus}, z=3 R_{\oplus}, r^{\prime}=\sqrt{r^{2}+z^{2}}=4.2 R_{\oplus}$ where $r$ is the horizontal distance from the planetary spin axis and $z$ is the vertical distance from the disk mid-plane, and $g=G M_{\oplus} z / r^{\prime 3}$. The choices of these parameters are discussed in Section 3.3). Assuming the surface area of the disk is $2 \pi\left(\left(5 R_{\oplus}\right)^{2}-R_{\oplus}^{2}\right)$ and the disk life time is 1000 years, the total amount of lost hydrogen is $5.86 \times 10^{14} \mathrm{~kg}$ and the equivalent amount of water is $5.86 \times 10^{14} \times(18 / 2)=5.27 \times 10^{15} \mathrm{~kg}$. It should be noted that this surface area is likely an upper limit because part the disk outside of the Roche radius would fragment. If the total mass of the disk is 1.5 lunar masses and the disk contains $100 \mathrm{ppm}$ of water, then the mass fraction of the lost water with respect to the total water (i.e., the water loss mass fraction) is $4.78 \times 10^{-4}$. This is too small to have a significant effect on the interpretation of measurements of the water abundance or $\mathrm{D} / \mathrm{H}$ ratio of the Moon.

Thus, determining the escape mechanism is highly important for estimating the volatile loss from the Moon-forming disk. In this paper, we determine the structure of the disk and find that the upper parts of the disk are dominated by heavy atoms and molecules (Sections 3.2 and 3.3). This indicates that the hydrogen escape is likely diffusion-limited and that the amount of hydrogen and other volatiles escaping from the disk is too small to be observed. This may indicate that the observed volatile loss (e.g., $\mathrm{K}, \mathrm{Na}$, and $\mathrm{Rb}$ ) would require another explanation as discussed in Section 4.2 .

\section{Model}

We assume that the disk has a liquid layer in the midplane that is sandwiched by vapor layers (this picture is similar to Figure 3 in Pahlevan and Stevenson 2007, but we assume that the disk is isolated from the Earth's atmosphere). We assume that the disk consists of water and silica $\left(\mathrm{SiO}_{2}\right.$ except Section 4.1, where $\mathrm{Mg}_{2} \mathrm{SiO}_{4}$ is considered). To estimate the hydrogen abundance in the upper parts of the disk, we simply investigate the vertical disk structure at a certain radial location (at $r=3 R_{\oplus}$ except Figure 4 given a mid-plane temperature $T_{\text {mid }}$ instead of modeling the whole disk structure. We make this simplification mainly because our outcome is insensitive to the detailed radial disk structure and partly because the time-dependent disk structure is not well known. The effect of $r$ on the water loss is considered in Section 3.3 .

The surface density of the disk is assumed to be $5 \times 10^{7} \mathrm{~kg} \mathrm{~m}^{-2}$ based on previous work (Canup et al., 2013; Nakajima and Stevenson, 2014). We also test $10^{8}$ $\mathrm{kg} \mathrm{m}^{-2}$ (not shown), but the outcome is similar to the 
case presented here. The model parameters are the bulk water content of the disk $(100,500$, and $1000 \mathrm{ppm})$ and mid-plane temperature $T_{\text {mid }}(2500-4000 \mathrm{~K})$. This range can be thought of as representing different stages in the cooling of the disk or different giant impact models (or both).

To estimate the amount of water loss, we first determine the mixing ratio of silicate vapor and water vapor in the upper parts of the disk without considering dissociation of molecules (Section 2.2). Based on the pressure and temperature ranges obtained, we estimate the mole fractions of the molecules and atoms considering dissociation given that the system in the chemical equilibrium (Section 2.3). If the disk is dominated by heavy elements, hydrogen escape is diffusion-limited. The amount of lost hydrogen and water is estimated using Equation (1).

\subsection{Boundary condition at the liquid-vapor interface}

At the liquid-vapor interface (at the mid-plane), the partial pressure of water is given as (Abe and Matsui, 1986)

$$
p_{\mathrm{H}_{2} \mathrm{O}}=\left(\frac{y_{\mathrm{H}_{2} \mathrm{O}}(\mathrm{wt} \%)}{2.08 \times 10^{-4}}\right)^{\frac{1}{0.54}}(\mathrm{~Pa}),
$$

where $y_{\mathrm{H}_{2} \mathrm{O}}$ is the mass fraction of water in the liquid. The saturation vapor pressure of pure $\mathrm{SiO}_{2}$ liquid $(2000-6000 \mathrm{~K})$ is written as (the units are modified from Visscher and Fegley 2013)

$$
p_{\mathrm{SiO}_{2}}^{*}=p_{0} \exp (-L / R T)
$$

where $p_{0}=1.596 \times 10^{13} \mathrm{~Pa}$ and $L=4.96 \times 10^{5} \mathrm{~J} \mathrm{~mol}^{-1}$. The total pressure at the interface becomes $p=(1-$ $\left.x_{\mathrm{H}_{2} \mathrm{O}}^{l}\right) p_{\mathrm{SiO}_{2}}^{*}+p_{\mathrm{H}_{2} \mathrm{O}}$, where $x_{\mathrm{H}_{2} \mathrm{O}}^{l}$ is the mole fraction of water in the liquid $\left(x_{\mathrm{H}_{2} \mathrm{O}}^{l}=\frac{y_{\mathrm{H}_{2} \mathrm{O}}}{18}\left(\frac{y_{\mathrm{H}_{2} \mathrm{O}}}{18}+\frac{100-y_{\mathrm{H}_{2} \mathrm{O}}}{60}\right)^{-1}\right)$.

\subsection{Vertical structure of the disk}

We assume that the disk is in the radiative-convective equilibrium, which implies that lower part of the disk (i.e., small $z$ ) is convective and the upper part (i.e., large $z$ ) is radiative. In previous studies, the Moon-forming disk is assumed to be convective (Thompson and Stevenson, 1988; Genda and Abe, 2003; Ward, 2012) or isothermal in the vertical direction (Charnoz and Michaut, 2015), but we modify this assumption in order to determine the temperature of the upper part of the disk. Provided the radial flow is highly subsonic, the disk is in hydrostatic equilibrium, i.e., $d p / d z=-\rho g$ where $p$ is the pressure, and $\rho$ is the density. The disk self-gravity is ignored.
The convective region of the disk follows the moist pseudoadiabat curve, which assumes that the partial pressure of silicate vapor is equal to its saturation vapor pressure. The amount of water extracted by solution in the silicate rain is small because of the modest water vapor pressure. This implies that only silicate rains out and this rain-out is efficient (if it were not then our conclusion that the water escape is minor would be even stronger since the upper atmosphere would contain droplets or particles that impede outflow). A moist pseudoadiabatic lapse rate is described as (Nakaiima et al., 1992),

$$
\left(\frac{\partial T}{\partial p}\right)_{s}=\frac{\frac{R T}{p C_{p, \mathrm{H}_{2} \mathrm{O}}}+\frac{x_{s}}{x_{\mathrm{H}_{2} \mathrm{O}} \mathrm{O}} \frac{L}{p C_{p, \mathrm{H}_{2} \mathrm{O}}}}{x_{\mathrm{H}_{2} \mathrm{O}}+x_{s} \frac{C_{p, s}}{C_{p, \mathrm{H}_{2} \mathrm{O}}}+\frac{x_{s}}{x_{\mathrm{H}_{2} \mathrm{O}} \mathrm{O}} \frac{L^{2}}{R T^{2} C_{p, \mathrm{H}_{2} \mathrm{O}}}},
$$

where $x_{s}$ is the mole fraction of saturated silicate (= $\left.p_{\mathrm{SiO}_{2}}^{*}(T) / p\right)$ and $x_{\mathrm{H}_{2} \mathrm{O}}\left(=1-x_{s}\right)$ is the mole fraction of water. The subscripts, $\mathrm{s}$ and $\mathrm{H}_{2} \mathrm{O}$ represent the parameters of $\mathrm{SiO}_{2}$ and water, respectively. It should be noted that dissociation of $\mathrm{SiO}_{2}$ and $\mathrm{H}_{2} \mathrm{O}$ are not considered in this section and it would be considered only in Section 2.3 The effect of this simplification on the temperature profile is further discussed in Section A1.2.$C_{p}$ is the specific heat and $C_{p, \mathrm{~s}}=62 \mathrm{JK}^{-1} \mathrm{~mol}^{-1}$, and $C_{p . \mathrm{H}, \mathrm{O}}=$ $55.7 \mathrm{JK}^{-1} \mathrm{~mol}^{-1}$ (at 1 bar at $3000 \mathrm{~K}$, Chase et al. 1985).

The optical depth throughout the disk is described as (Nakajima et al., 1992)

$$
d \tau=\left(\kappa_{s} x_{s} m_{s}+\kappa_{\mathrm{H}_{2} \mathrm{O}} x_{\mathrm{H}_{2} \mathrm{O}} m_{\mathrm{H}_{2} \mathrm{O}}\right) \frac{d p}{m_{\mathrm{ave}} g},
$$

where $\tau$ is the optical depth, $\kappa$ is the absorption coefficient, $m$ is the molecular weight, and $m_{\text {ave }}$ is the average molecular weight $\left(m_{s}=m_{\mathrm{SiO}_{2}}=60 \mathrm{~g} \mathrm{~mol}^{-1}\right.$ and $\left.m_{\mathrm{H}, \mathrm{O}}=18 \mathrm{~g} \mathrm{~mol}^{-1}\right)$. Here, $\kappa_{s}=0.1 \mathrm{~m}^{2} \mathrm{~kg}^{-1}$ (Thompson and Stevenson, 1988) and $\kappa_{\mathrm{H}_{2} \mathrm{O}}=0.01$ $\mathrm{m}^{2} \mathrm{~kg}^{-1}$ are used (Nakajima et al., 1992). We assume that the disk is treated as a gray atmosphere and the upward radiation flux at given $\tau$ is written as

$$
\begin{aligned}
F_{\uparrow}(\tau) & =\frac{3}{2} \int_{\tau}^{\tau_{\text {mid }}} \pi B\left(\tau^{\prime}\right) \exp \left[-\frac{3}{2}\left(\tau^{\prime}-\tau\right)\right] d \tau^{\prime} \\
& +\pi B\left(\tau_{\text {mid }}\right) \exp \left[\frac{3}{2}\left(\tau-\tau_{\text {mid }}\right)\right],
\end{aligned}
$$

where $\tau_{\text {mid }}$ is the optical depth at the mid-plane (the liquid-vapor boundary). Likewise, the downward radiation flux is written as

$$
F_{\downarrow}(\tau)=\frac{3}{2} \int_{0}^{\tau} \pi B\left(\tau^{\prime}\right) \exp \left[-\frac{3}{2}\left(\tau^{\prime}-\tau\right)\right] d \tau^{\prime} .
$$


The net upward flux is written as $F(\tau)=F_{\uparrow}(\tau)-F_{\downarrow}(\tau)$. Here, $\pi B=\sigma T^{4}$ where $\sigma$ is the Stefan-Boltzmann constant. In the radiative part of the disk, the temperature profile follows the relationship

$$
\pi B=\sigma T^{4}(\tau)=\frac{1}{2} F_{\uparrow \text { top }}\left(\frac{3}{2} \tau+1\right)
$$

where $F_{\uparrow t o p}$ is the radiation flux from the top of the atmosphere (where $\tau=0$ ). The transition (tropopause) between the convective lower disk and radiative upper disk is iteratively determined. First, assuming that the whole atmosphere is convective, we compute the vertical structure of the disk until the condition described by Equation (10) is met. This provides $\tau_{\text {tp }}, T_{\text {tp }}$, and $F_{\text {tp }}$ where $F_{\text {tp }}=F_{\uparrow \text { top }}$. We recalculate Equations (8) and (9) and adjust the value of $F_{\uparrow \text { top }}$ assuming that the lower parts of the disk are convective and the upper parts of the disk are radiative (generally speaking, this correction is very minor).

\subsection{Dissociation of molecules}

At high temperatures and low pressures, molecules in the disk can dissociate. For example, assuming that the system is in the chemical equilibrium, the dissociation of $\mathrm{SiO}_{2}=\mathrm{SiO}+\mathrm{O}$ is described as

$$
K_{\text {th }}=\frac{p_{\mathrm{SiO}_{\mathrm{O}}} p_{\mathrm{O}}}{p_{\mathrm{SiO}_{2}}}=\exp \left(-\Delta G^{0} / R T\right),
$$

where $K_{\mathrm{th}}$ is the equilibrium constant, and $p_{\mathrm{SiO}}, p_{\mathrm{O}}$, and $p_{\mathrm{SiO}_{2}}$ are the partial pressures of $\mathrm{SiO}, \mathrm{O}$ and $\mathrm{SiO}_{2}$ in bar. $G^{0}$ is the Gibbs free energy under the standard conditions $\left(\Delta G^{0}=\Delta H^{0}-T \Delta S^{0}\right)$. Here, $\Delta H^{0}$ is the change in the Helmholtz energy and $\Delta S^{0}$ is the change in the entropy. We assume that $\Delta H^{0}$ and $\Delta S^{0}$ are not sensitive to temperature and pressure. The rest of the reactions and the thermal constants are listed in Table 1 .

\subsection{Homopause location}

The escape flux of hydrogen is determined by thermal properties at the homopause, where eddy diffusion $K$ equals molecular diffusion $D$ (the molecular diffusion coefficient is described as $D=b_{i j} / N$ where $N$ is the number density). At lower parts of the disk (small $z$ ), eddy diffusion is more efficient than molecular diffusion $(K>D)$, and therefore the disk is homogenized (i.e., the mole fractions of atoms and molecules are constant). On the other hand, in the upper parts of the disk (large $z$ ), molecular diffusion becomes more dominant $(K<D)$, and therefore each molecule or atom has its own scale height (i.e. light elements are more abundant at large $z$ ). If a hydrogen atom (or any light element) is present above the homopause, the atom can easily escape from the disk. However, this escape rate cannot exceed the hydrogen supply below the homopause. In other words, the supply rate is determined by how fast a hydrogen atom can diffuse from a heavy-element rich disk. This is the definition of diffusion-limited escape as we briefly describe in Section 1 We estimate that the homopause pressure range is $\sim 10^{-4}-10^{1} \mathrm{~Pa}$, as we discuss in Section A1.1. Even though the pressure range of homopause is not well defined, fortunately, our result is insensitive to this parameter.

\section{Results}

\subsection{Vertical structure of the disk}

The vertical structure of the disk is shown in Figure 1. The left panels show the temperature-pressure structure and right panels show the mixing ratio of water $\left(x_{\mathrm{H}_{2} \mathrm{O}}\right)$. The top, middle, and bottom panels correspond to the cases when the bulk water abundance is (a) $100 \mathrm{ppm}$, (b) $500 \mathrm{ppm}$, and (c) $1000 \mathrm{ppm}$. The location of the homopause is indicated by the shade (a large uncertainty, as discussed in Section 2.4 and A1.1. The lower parts of the disk (i.e., large $p$ and small $z$ ) are in the convection regime while the upper parts (i.e., small $p$ and small $z$ ) are in the radiative regime. The location of this transition (i.e., tropopause) is indicated by the kink in the temperature profile. This kink appears because the disk temperature continues to decrease in the convective regime as $z$ increases, whereas it is nearly constant in the radiative regime.

The water mixing ratio at the homopause is most sensitive to the mid-plane temperature. As Figure 1 a2-c2 shows, at high mid-plane temperatures $\left(T_{\text {mid }}>2500\right.$ $2800 \mathrm{~K}$ ) the homopause is dominated by silicate vapor $\left(x_{\mathrm{H}_{2} \mathrm{O}}<0.5\right)$ because the partial pressure of the silicate vapor is larger than that of water vapor. In contrast, it is dominated by water vapor $\left(x_{\mathrm{H}_{2} \mathrm{O}}>0.5\right)$ at lower mid-plane temperatures $\left(T_{\text {mid }}<2500-2800 \mathrm{~K}\right)$ because most of the silicate condenses into liquid and the partial pressure of silicate vapor becomes negligible. As the bulk water abundance increases, the water mixing ratio increases, but this is a relatively weak effect compared to the mid-plane temperature.

When the disk is dominated by silicate vapor, the temperature of the disk closely follows the saturation vapor pressure of $\mathrm{SiO}_{2}$ (i.e., it is thermodynamically determined rather than determined by the heat flow). This is a universal curve (independent of disk mass, heat 


\begin{tabular}{|ccc|}
\hline Reaction & $\Delta S^{o}(\mathrm{~J} / \mathrm{mol} \mathrm{K})$ & $\Delta H^{o}(\mathrm{~kJ} / \mathrm{mol})$ \\
\hline $\mathrm{SiO}_{2}=\mathrm{SiO}+\frac{1}{2} \mathrm{O}_{2}$ & 85.2 & 205 \\
$\mathrm{SiO}=\mathrm{Si}+\frac{1}{2} \mathrm{O}_{2}$ & 85.1 & 550 \\
$\mathrm{MgO}=\mathrm{Mg}+\frac{1}{2} \mathrm{O}_{2}$ & 38.0 & 88 \\
$\mathrm{O}_{2}=2 \mathrm{O}$ & 117 & 498 \\
$\mathrm{H}_{2} \mathrm{O}=\mathrm{H}_{2}+\frac{1}{2} \mathrm{O}_{2}$ & 44.4 & 242 \\
$\mathrm{H}_{2}=2 \mathrm{H}$ & 98.8 & 436 \\
\hline
\end{tabular}

Table 1: Reactions and thermodynamic constants (Chase et al., 1985). All the elements are in the vapor phase.

flow, and water provided $\left.x_{\mathrm{H}_{2} \mathrm{O}} \ll 1\right)$. In contrast, when the disk is dominated by water $\left(x_{\mathrm{H}_{2} \mathrm{O}} \sim 1\right)$, the disk temperature profile is determined by the heat flow (i.e., the radiative boundary condition) and the temperature can be less than the value it would have if it were determined by vapor pressure equilibrium with silicate because there is no longer significant silicate present. The homopause height is $z \sim 3 R_{\oplus}$ for a silicate-rich disk, whereas $z \sim 5 R_{\oplus}$ for a water-rich disk.

\subsection{Atoms and molecules present in the disk}

The atoms and molecules present at the homopause in the disk at a constant temperature are shown in Figures 2 and 3 In Figure 2 (a) and (b), the water mixing ratio $x_{\mathrm{H}_{2} \mathrm{O}}$ is 0.1 and 0.5 , respectively while $x_{\mathrm{H}_{2} \mathrm{O}}$ is 1 in Figure 3. Figure 2 corresponds to cases where the midplane temperature is high (i.e. $T_{\text {mid }}>2500-2800 \mathrm{~K}$ ) and the disk is dominated by silicate vapor, while Figure 3 corresponds to the cases where the mid-plane temperature is relatively small (i.e. $T_{\text {mid }}<2500-2800 \mathrm{~K}$ ) and the homopause is dominated by water vapor.

In Figure 2 (a), the main reactions are described as $\mathrm{SiO}_{2}=\mathrm{SiO}+\mathrm{O}$ and $\mathrm{H}_{2} \mathrm{O}=2 \mathrm{H}+\mathrm{O}$ at $p<10^{-3} \mathrm{~Pa}$. Hydrogen mole fraction $f_{\mathrm{H}}$ at this pressure range is $\sim 0.1$ (this is approximately estimated by $0.9 \mathrm{SiO}_{2}+0.1 \mathrm{H}_{2} \mathrm{O}$ $=0.9 \mathrm{SiO}+1 \mathrm{O}+0.2 \mathrm{H} . f_{\mathrm{H}} \sim 0.2 /(0.9+1+0.2)=$ 0.095). A similar argument can be made for Figure 2 (b), where $0.5 \mathrm{SiO}_{2}+0.5 \mathrm{H}_{2} \mathrm{O}=0.5 \mathrm{SiO}+1 \mathrm{O}+1 \mathrm{H}$, therefore $f_{\mathrm{H}}=1 /(0.5+2)=0.4$. This $f_{\mathrm{H}}$ is likely to be an overestimate because the homopause temperature is below $2000 \mathrm{~K}$ at $x_{\mathrm{H}_{2} \mathrm{O}}=0.5$ (for example, the homopause temperature is $1660 \mathrm{~K}$ at $T_{\text {mid }}=2500 \mathrm{~K}$ and $p=10^{-2} \mathrm{~Pa}$ with $100 \mathrm{ppm}$ of water as shown in a1, Figure 1). $x_{\mathrm{H}_{2} \mathrm{O}}>0.5$ can occur when the water abundance is $1000 \mathrm{ppm}\left(\mathrm{c} 2\right.$, Figure 1 at $\left.T_{\text {mid }}=2800 \mathrm{~K}\right)$, but $1000 \mathrm{ppm}$ is likely to be larger than the actual bulk lunar water abundance (Section 1 .

When the homopause is dominated by water $\left(x_{\mathrm{H}_{2} \mathrm{O}} \sim\right.$ 1), the homopause temperature is approximately 1600 $\mathrm{K}$ or below at $p<1(\mathrm{~Pa})$ (Figure 1). Under these circumstances, $\mathrm{H}_{2} \mathrm{O}$ exists as its molecular form, and thus, hydrogen $\mathrm{H}$ is not abundant due to this relatively low temperature as shown in Figure 3 (a). If the homopause temperature is close to $2000 \mathrm{~K}$ (Figure 3b), $\mathrm{H}_{2} \mathrm{O}$ is dissociated to $2 \mathrm{H}$ and $\mathrm{O}$, and hydrodynamic escape would be expected from such a disk as suggested by Desch and Taylor (2013). However, we suggest that this is unlikely to be the case for the lunar disk because the homopause temperature is smaller $(\sim 1600 \mathrm{~K})$.

\subsection{Water loss from the disk}

Hydrogen loss from the disk is estimated from Equation (1). First, we consider the case when the disk temperature is high and the disk is dominated by silicate vapor $\left(f_{\mathrm{H}}<0.4\right.$ as discussed in Section 3.2). This is likely the case shortly after the Moon-forming impact when the disk temperature is close to the initial disk temperature (4000-6000 K, Nakajima and Stevenson 2014). Assuming the disk is dominated by $\mathrm{O}$ with a minor amount of $\mathrm{H}\left(f_{\mathrm{H}}=0.1, f_{\mathrm{O}}=0.9\right)$, the lost water by diffusionlimited escape is estimated as $5.27 \times 10^{15} \mathrm{~kg}$ and the mass fraction of lost lunar water is $4.78 \times 10^{-4}$ as discussed in Section 1 .

Now we consider the case when the homopause is dominated by water. Given the homopause temperature is $1600 \mathrm{~K}, f_{\mathrm{H}}=0.3, m_{2}=18 \mathrm{~g} \mathrm{~mol}^{-1}, \sigma_{2}=265 \mathrm{pm}, z=$ $5 R_{\oplus}$ with bulk $100 \mathrm{ppm}$ of water, the hydrogen escape flux is $1.64 \times 10^{15}$ atoms $\mathrm{m}^{-2} \mathrm{~s}^{-1}$, the lost water mass is $4.73 \times 10^{15} \mathrm{~kg}$ and the mass fraction of lost water is $4.28 \times 10^{-4}$.

The mass fraction of water loss with various parameters $\left(T=1600 \mathrm{~K}\right.$ and $2000 \mathrm{~K}, f_{\mathrm{H}}=0.1,0.3$, and $\left.0.5, r=3-7 R_{\oplus}\right)$ is shown in Figure 4 , which confirms that water loss from the disk is minor $(<$ a few $10^{-3}$ under these conditions). The range of $r$ is taken from previous work $\left(r \leq 5-7 R_{\oplus}\right.$, Canup et al. 2013; Nakaijima and Stevenson 2014).

\section{Discussion}

\section{1. $\mathrm{Mg}_{2} \mathrm{SiO}_{4}$ disk}

In Section 3, we only consider a disk that contains $\mathrm{SiO}_{2}$ and $\mathrm{H}_{2} \mathrm{O}$, but the disk composition would be 

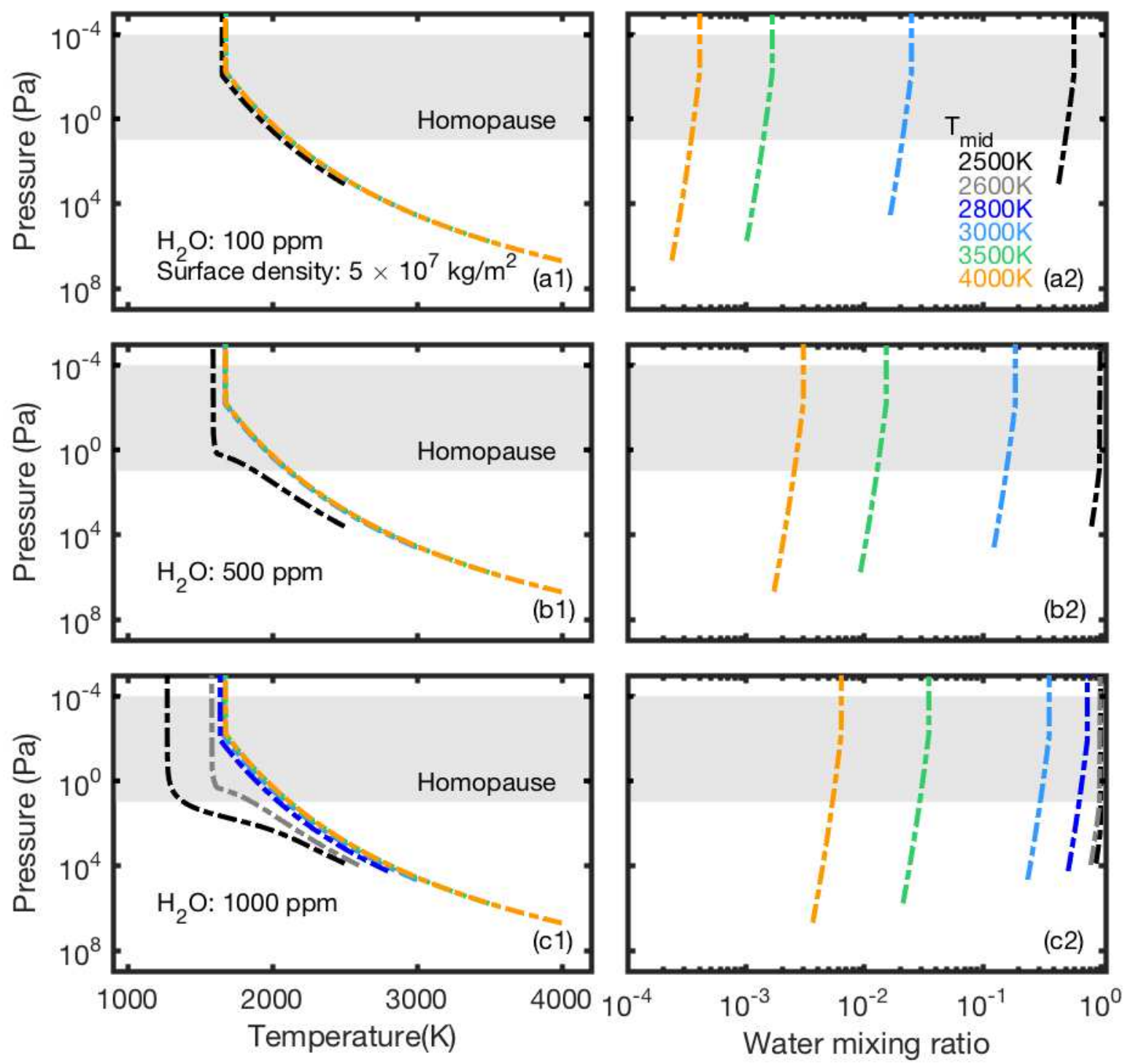

Figure 1: Vertical structure of the Moon-forming disk with the surface density of $5 \times 10^{7} \mathrm{~kg} \mathrm{~m}^{-2}$. The top, middle, and bottom panels represent cases when the bulk water abundance is $100 \mathrm{ppm}, 500 \mathrm{ppm}$, and $1000 \mathrm{ppm}$, respectively. The black, gray, blue, sky-blue, green, and orange lines correspond to $T_{\text {mid }}=2500 \mathrm{~K}, 2600 \mathrm{~K}, 2800 \mathrm{~K}, 3000 \mathrm{~K}, 3500 \mathrm{~K}$, and $4000 \mathrm{~K}(2600 \mathrm{~K}$ and $2800 \mathrm{~K}$ are shown only for the case with 1000 ppm water). The homopause location is indicated by the shade. The left panels show the temperature whereas the right panels show the water mixing ratio $\left(x_{\mathrm{H}_{2} \mathrm{O}}\right)$ as a function of pressure. When the disk temperature is high and the bulk water abundance is low, the water mixing ratio is small and the pressure closely follows the saturation vapor pressure of $\mathrm{SiO}_{2}$. In contrast, when the disk temperature is small, the upper disk atmosphere is dominated by water and the pressure is smaller than the saturated vapor pressure of $\mathrm{SiO}_{2}$. 


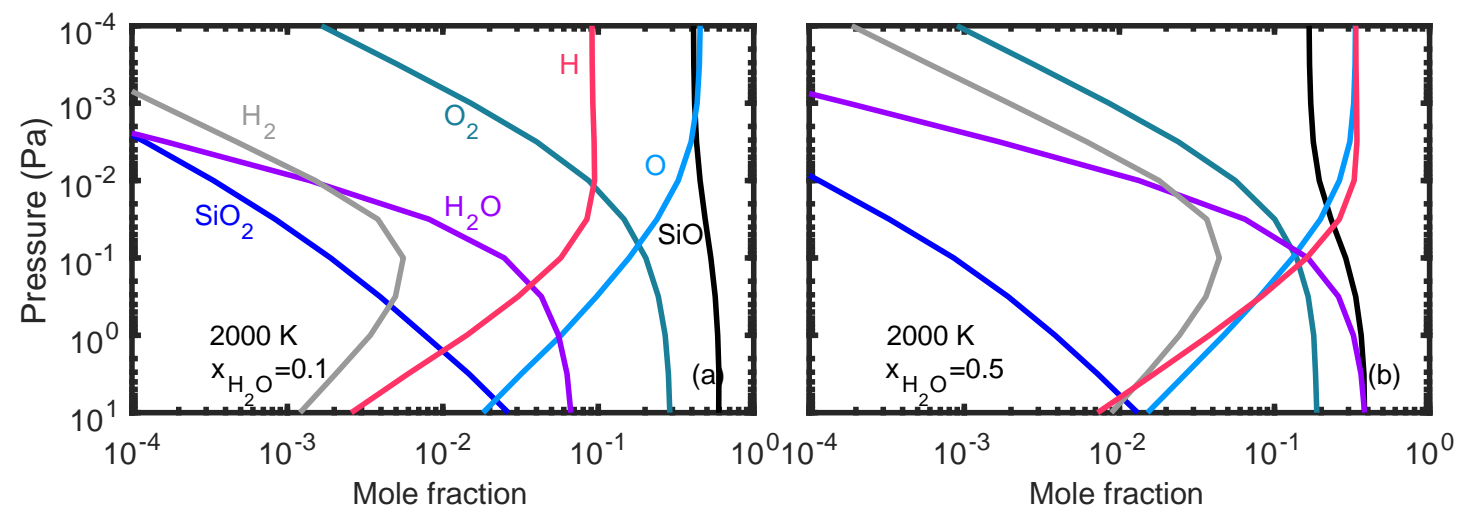

Figure 2: Species that are present at the homopause at $2000 \mathrm{~K}$. The y-axis corresponds to the homopause pressure (Section 2.4 $. \mathrm{SiO}_{2}, \mathrm{SiO}_{2} \mathrm{O}_{2}, \mathrm{O}$, $\mathrm{H}_{2} \mathrm{O}, \mathrm{H}_{2}$, and $\mathrm{H}$ are shown in blue, black, green, sky blue, purple, gray, and magenta (the Si mole fraction is too small to be shown in this figure). The left panel shows the dissociation of $\mathrm{SiO}_{2}$ at $x_{\mathrm{H}_{2} \mathrm{O}}=0.1$ and the right panel shows the case at $x_{\mathrm{H}_{2} \mathrm{O}}=0.5$. In both cases, the hydrogen mole fraction $f_{\mathrm{H}}$ is small $(<0.4)$.

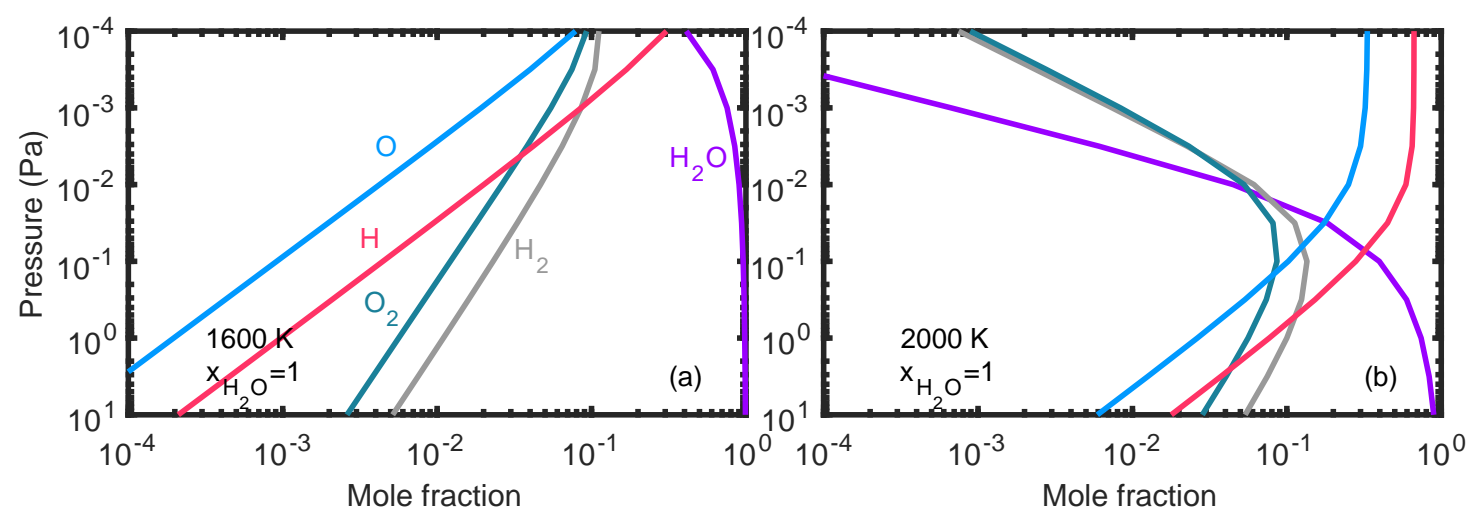

Figure 3: Dissociation of pure $\mathrm{H}_{2} \mathrm{O}$ at $1600 \mathrm{~K}$ (left) and $2000 \mathrm{~K}$ (right). At $1600 \mathrm{~K}, \mathrm{H}_{2} \mathrm{O}$ is the dominant species, whereas $\mathrm{H}$ is dominant at low pressures at $2000 \mathrm{~K}$. The left panel $(1600 \mathrm{~K})$ better describes the Moon-forming disk because the homopause temperature is close to $1600 \mathrm{~K}$ when the homopause is dominated by water. 


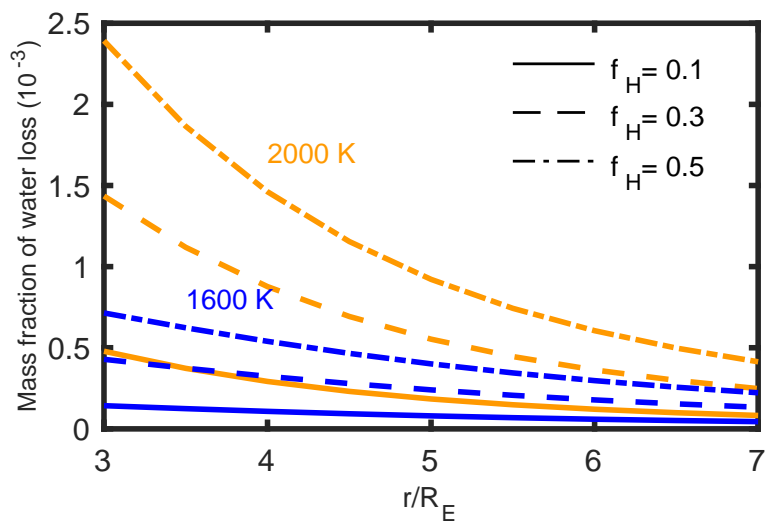

Figure 4: Mass fraction of water loss with the various disk temperatures and the hydrogen mole fractions. The solid line, dashed line, and dotdash lines correspond to $f_{\mathrm{H}}=0.1,0.3$, and 0.5 . The blue and orange lines represent $1600 \mathrm{~K}$ and $2000 \mathrm{~K}$, respectively. For this calculation, we assume that the disk is dominated by $\mathrm{H}_{2} \mathrm{O}$ at $1600 \mathrm{~K}$ and by $\mathrm{O}$ at $2000 \mathrm{~K}$, as discussed in Sections 1 and 3.3

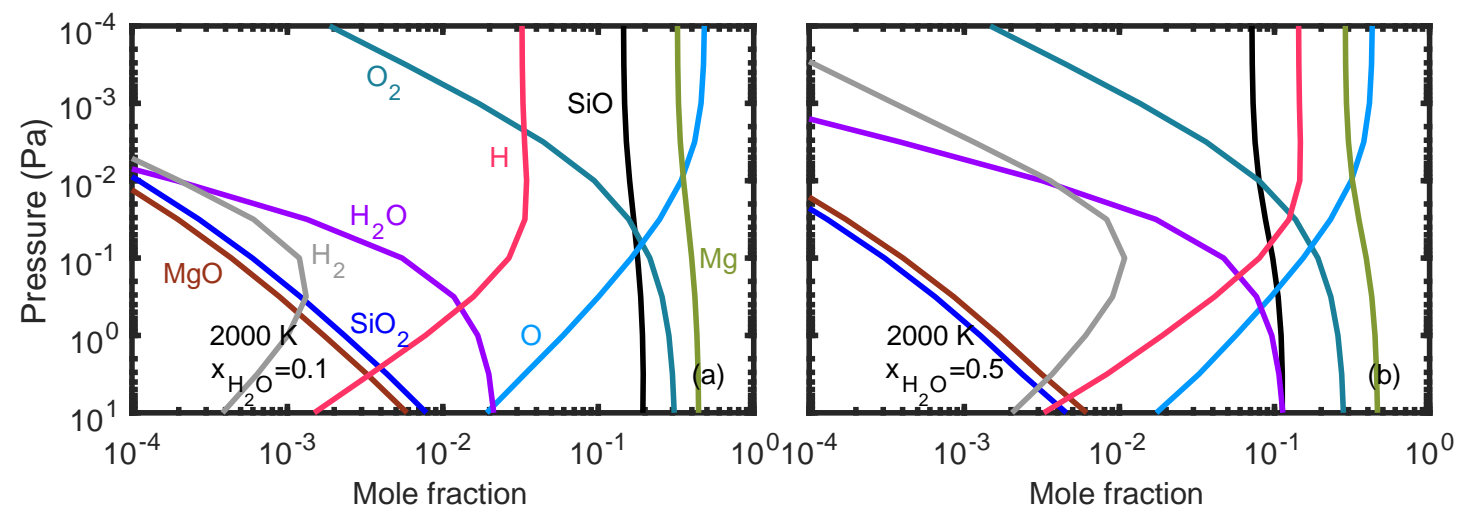

Figure 5: Dissociation of $\mathrm{Mg}_{2} \mathrm{SiO}_{4}$ and $\mathrm{H}_{2} \mathrm{O}$. The color scheme is the same as Figure2 with additional elements (MgO and $\mathrm{Mg}$ are shown in brown and green). The hydrogen mole fraction is smaller than the case of $\mathrm{SiO}_{2}$ because additional species (mainly $\mathrm{Mg}$ and $\mathrm{O}$ ) are produced. 
better modeled by $\mathrm{Mg}_{2} \mathrm{SiO}_{4}$. The disk temperaturepressure profile would not be significantly different given that the saturation vapor pressure of the bulk silicate Earth composition is similar to that of $\mathrm{SiO}_{2}$ (Figure 3, Visscher and Fegley 2013), whereas species in the disk would be different. Figure 5 shows the mole fractions of the species present in a disk of $\mathrm{Mg}_{2} \mathrm{SiO}_{4}$ and $\mathrm{H}_{2} \mathrm{O}$. At $x_{\mathrm{H}_{2} \mathrm{O}}=0.5$, the main reactions are described as $0.5 \mathrm{Mg}_{2} \mathrm{SiO}_{4}=0.5(2 \mathrm{Mg}+\mathrm{SiO}+3 \mathrm{O})$ and $0.5 \mathrm{H}_{2} \mathrm{O}=0.5(2 \mathrm{H}+\mathrm{O})$, which leads to $f_{\mathrm{H}}=0.22$. The presence of $\mathrm{Mg}$ decreases $f_{\mathrm{H}}$ and makes it even more difficult to lose hydrogen from the disk. $f_{\mathrm{H}}$ may decrease even further when more species (such as $\mathrm{Al}_{2} \mathrm{O}_{3}$ and $\mathrm{CaO}$ ) that are expected to be in the disk are considered (e.g., Visscher and Fegley, 2013; Ito et al., 2015). We do not consider effects of $\mathrm{Mg}$ on the solubility of water in the magma even though it could dissolve as $\mathrm{Mg}(\mathrm{OH})_{2}$ (for detailed discussion, see Fegley et al. 2016). Furthermore, some $\mathrm{OH}$ can be present in the disk (Pahlevan et al.2016 and personal communications with $\mathrm{J}$. Melosh), which is not considered in this paper. It is possible that presence of $\mathrm{OH}$ can decrease $f_{\mathrm{H}}$ and the amount of water loss.

\subsection{Escape of other volatiles}

In the previous sections, we only consider the escape of hydrogen, but other volatile elements, such as $\mathrm{K}$ and $\mathrm{Na}$, can be considered in a similar framework. Zahnle et al. (1990) consider the escape of a minor species with the presence of two major constituents. The escape flux of a minor constituent $k, \phi_{k}$, is approximately described as,

$$
\phi_{k}=F_{k} \phi_{\mathrm{H}}\left(\frac{1-\frac{m_{k}-m_{\mathrm{H}}}{m_{2}-m_{\mathrm{H}}} \frac{b_{\mathrm{H} k}}{b_{\mathrm{H} 2}}+\frac{m_{2}-m_{k}}{m_{2}-m_{\mathrm{H}}} \frac{b_{\mathrm{H} k}}{b_{\mathrm{H} 2}} F_{2}}{1+\frac{b_{\mathrm{H} k}}{b_{2 k}} F_{2}}\right),
$$

where the subscripts $\mathrm{H}, 2$, and $k$ represent hydrogen, heavy element that does not escape (corresponding to species such as $\mathrm{SiO}$ and $\mathrm{O}$ in the Moon-forming disk), and the element $k$, respectively. Here, $F_{k}=\frac{n_{k}}{n_{H}}$ and $F_{2}=\frac{n_{2}}{n_{H}}$ (the definition of $F_{k}$ is slightly different from $f_{k}=\frac{n_{H_{k}}}{n_{H}+n_{i}+n_{k}}$ as described in Section 10.

Assumptions of this model are (1) $F_{k} \ll F_{2}, 1$, and (2) $F_{2}$ is small. If the element $k$ is potassium and the element 2 is atomic oxygen, given that $\sigma_{2}=2 \times 60$ pm, $\sigma_{k}=2 \times 220 \mathrm{pm}, m_{2}=16 \mathrm{~g} \mathrm{~mol}^{-1}, m_{k}=39.1$ $\mathrm{g} \mathrm{mol}^{-1}$, and $F_{2}=1$, and the equation above becomes $\phi_{k}=0.0683 F_{k} \phi_{\mathrm{H}}$. Assuming that $F_{\mathrm{k}}=1$, and $\phi_{H} \sim 2 \times 10^{15}$ atoms $\mathrm{m}^{-2} \mathrm{~s}^{-1}, \phi_{k}=1.37 \times 10^{14}$ atoms $\mathrm{m}^{-2} \mathrm{~s}^{-1}$, which corresponds to $1.73 \times 10^{15} \mathrm{~kg}$. This is much smaller than the total potassium mass of the
Moon is $2.64 \times 10^{19} \mathrm{~kg}$ if the potassium abundance of the Moon-forming disk (1.5 lunar masses) is the same as terrestrial values (240 ppm, McDonough and Sun 1995). Equation (12) may not necessarily be useful for estimating other volatiles such as $\mathrm{Na}$, because $\mathrm{Na}$ may be a dominant constituent instead of a minor constituent (Visscher and Fegley, 2013). However, it is still unlikely that this weak hydrogen flux would drag other heavier volatile elements.

Given that diffusion-limited escape is not an efficient mechanism for removing water or volatiles from the Moon-forming disk, the next question becomes how the Moon lost its volatiles or failed to efficiently accrete the volatiles. A potential explanation is to accrete these volatiles onto Earth. Canup et al. (2015) suggest that some volatiles that were initially present in the disk could have been preferentially accreted onto the Earth at the end of the Moon accretion process. The amount is not a significant part of the total volatile budget of Earth and may only have a small effect on the isotopic ratios for the Moon because high disk temperature may not cause significant isotopic fractionation especially if it is described as equilibrium isotope fractionation. Slightly higher lunar potassium isotopic ratios than those of the Earth may also indicate that the Moon forms from liquid part of the disk (Wang and Jacobsen, 2016; Lock et al., 2016). An alternative is to lose volatiles directly from the Moon as or after it accretes (Section 4.5).

\subsection{Model validation}

First, in Section 3, we assume that heavy elements, such as oxygen O, would not escape from the disk, but this is an approximation. Some oxygen would escape, but the amount is likely to be limited. The escape regime of oxygen would be diffusion-limited because $\mathrm{O}$ would be too heavy to hydrodynamically escape. Consider a disk that consists of $\mathrm{SiO}$ and $\mathrm{O}$, and $f_{\mathrm{O}}=0.5$, $T=2000 \mathrm{~K}, \sigma_{1}=2 \times 60 \mathrm{pm}, \sigma_{2}=320 \mathrm{pm}, z=3 R_{\oplus}$ then the escape flux of $\mathrm{O}$ becomes $1.88 \times 10^{15}$ molecules $\mathrm{m}^{-2} \mathrm{~s}^{-1}$. This is too small to affect the oxygen abundance in the Moon.

For the two cases discussed in Section 3.3 (silicaterich and water-rich disks), the Jeans parameter $\lambda=$ $G M_{\oplus} \bar{m} / R T r^{\prime}$ is estimated as $26.9\left(\bar{m}=30 \mathrm{~g} \mathrm{~mol}^{-1}\right.$, $T=2000 \mathrm{~K}$, and $\left.r^{\prime}=4.2 R_{\oplus}\right)$ and $14.6(\bar{m}=18 \mathrm{~g}$ $\mathrm{mol}^{-1}, T=1600 \mathrm{~K}$, and $\left.r^{\prime}=5.8 R_{\oplus}\right)$, both of which are much smaller than 2 , suggesting that hydrogen escape is diffusion-limited. It should be noted, however, that the criterion (i.e., hydrodynamic escape at $\lambda<2$ ) is developed for an isotropic atmosphere with a single component, and therefore it may not be directly applicable to a Moon-forming disk which is neither isotropic 
nor a single component. Nevertheless, $\lambda$ being much larger than 2 further supports our model that the escape is diffusion-limited.

Furthermore, we use the formula of diffusion-limited escape developed for spherical geometry, but this geometry is not exactly applicable to the Moon-forming disk. Nevertheless, we argue that the geometry becomes similar to a sphere given that the disk is extended in the vertical direction $\left(z \sim 3-5 R_{\oplus}\right)$. Furthermore, the physics of diffusion-limited escape is not sensitive to the geometry. We have not explicitly modeled the non-thermal aspect of the disk evolution, such as the decrease in surface density as disk material spreads outward as well as disk materials accreting to the Moon and Earth simultaneously. However, much of this effect is simply equivalent to changing surface density and the results are not sensitive to this. Of course, aggregating material at large radius or onto Earth can only decrease the escape. Moreover, here we assume that the disk is isolated, but if the interaction of the disk and atmosphere is considered, the escape may become even smaller because volatiles in the disk may be replenished from Earth.

In this paper, we focus on diffusion-limited escape, but volatile escape can be limited by other sources. Some of the previous studies investigate energy-limited escape, where the escape is caused by energy input from the stellar EUV, and therefore the rate is limited by the stellar flux. In that case, the escape rate is written as $\phi_{\mathrm{EL}}=\frac{\varepsilon F^{*} r^{\prime}}{G M m^{\prime}}$, where $\varepsilon$ is the efficiency factor, $m^{\prime}$ is the averaged molecular weight, $F^{*}$ is the globally averaged EUV flux and Lyman $\alpha$ flux, which could have been $10^{-2}-10^{-1} \mathrm{~W}$ $\mathrm{m}^{-2}$ during this time period (e.g., Zahnle et al., 1990; Baraffe et al., 2004; Genda and Ikoma, 2008). At $\varepsilon=$ 0.3 (Murray-Clay et al., 2009), $m^{\prime}=12.9 \mathrm{~g} \mathrm{~mol}^{-1}$ (mixture of $0.3 \mathrm{H}$ and $\left.0.7 \mathrm{H}_{2} \mathrm{O}\right), F^{*}=0.1 \mathrm{Wm}^{-2}, r^{\prime}=4.2 R_{\oplus}$, this becomes $9.41 \times 10^{16}$ atoms $\mathrm{m}^{-2} \mathrm{~s}^{-1}$ assuming the same disk surface area and disk life time discussed in Section 1. This value is much larger than the diffusionlimited case. However, this is equivalent to losing $4.61 \times 10^{17} \mathrm{~kg}$ of water, which is still relatively small compared to the lunar bulk water $\left(1.10 \times 10^{19} \mathrm{~kg}\right.$ at 100 ppm). This energy-limited escape can be more efficient if the escape occurs at higher $z$, where $f_{\mathrm{H}}$ is larger and $m^{\prime}$ is smaller, than the homopause, but then the rate would be ultimately limited by the hydrogen supply from below, and therefore the escape rate may not exceed the diffusion-limited escape rate. Thus, it is possible that energy-limited escape would contribute to water loss, but the extent is likely to be limited. We further discuss model assumptions in Section A1.2.

\subsection{Comparison among different impact models}

Our calculations show that hydrogen escape is minor at $T_{\text {mid }}=2500-4000 \mathrm{~K}$, where $4000 \mathrm{~K}$ is the initial disk temperature estimated for the canonical Moonforming impact. $T_{\text {mid }}$ can be higher than $4000 \mathrm{~K}$ in the fast-spinning Earth and half-Earths models $\left(T_{\text {mid }}=\right.$ $6000-7000$ K, Nakajima and Stevenson 2014). Nevertheless, even under this temperature rage, escape is still inefficient because $f_{\mathrm{H}}$ at the homopause is small. Thus, water escape would be inefficient even outside of the temperature range considered in this paper.

\subsection{Lunar volatiles after the Moon formation}

In this paper, we focus on volatile loss during the disk phase, and it is possible that some volatiles were lost or added before and after this phase. For example, the giant impact may have induced a vapor jet (Melosh and Sonnet, 1986; Karato, 2014), which may have removed some volatiles from the Moon-forming disk. This extent is difficult to quantify with the conventional impact method called smoothed particle hydrodynamics (SPH), where a fluid is expressed as a collection of spherical particles. This is because the current SPH method cannot treat physics of twophase (liquid-vapor) flow. Furthermore, some volatiles may have been added to or removed from the Moon before the lunar crust formation (Bottke et al., 2010; Elkins-Tanton et al., 2011; Elkins-Tanton and Grove, 2011; Sharp et al., 2013; Hauri et al., 2015, 2017), which depends on the impact flux and crust formation time scale $\left(\sim 10-10^{2}\right.$ Myrs, Meyer et al. 2010; Elkins-Tanton et al. 2011). We will further investigate evolution of lunar volatiles in the future.

\section{Conclusions}

We estimate the upper limit for hydrogen and volatile loss by thermal escape from the Moon-forming disk under various disk mid-plane temperatures (2500 - 4000 $\mathrm{K})$ and the bulk water abundances (100, 500 and 1000 $\mathrm{ppm})$. When the mid-plane disk temperature is large $(>2500-2800 \mathrm{~K})$, the disk is dominated by silicate vapor. The major species in the upper part of the disk are $\mathrm{SiO}$ and $\mathrm{O}$ and the hydrogen mole fraction is small. In contrast, under low disk mid-plane temperature $(<2500-2800 \mathrm{~K})$, the upper part of the disk is dominated by water and its temperature is $\sim 1600 \mathrm{~K}$ or below. In this temperature range, water stays in its molecular form $\left(\mathrm{H}_{2} \mathrm{O}\right)$ and the hydrogen mole fraction is small as well. Since hydrogen is not the major element and other heavy elements (such as $\mathrm{O}, \mathrm{SiO}, \mathrm{SiO}_{2}$, and 
$\mathrm{H}_{2} \mathrm{O}$ ) are the dominant species, hydrogen would need to diffuse out from this heavy-element rich disk. This escape regime is called diffusion-limited escape and it is an inefficient escape process. We estimate the total mass of lost water and volatiles, such as potassium, and find that the escape is inefficient and that it would not remove water or other volatiles from the disk to the extent that the loss is measurable in lunar rock samples. To remove volatiles from the Moon-forming disk or from the Moon, another mechanism, such as losing volatiles from the disk to the Earth or degassing from the lunar surface, would be required.

\section{Acknowledgement}

This work is supported by NASA Headquarters under the NASA Earth and Space Science Fellowship Program Grant NNX14AP26H and the Carnegie DTM Postdoctoral Fellowship. We would like to thank Masahiro Ikoma, Francis Nimmo, Cheng Li, Jay Melosh, Steve Desch, Erik Hauri, Alycia Weinberger, Peng Ni, and anonymous reviewers for helpful discussions.

\section{Supplementary Information}

\section{A1.1. Homopause location}

The location of the homopause is defined where $K=D$. The value of $K$ is highly uncertain because it is determined by dynamical processes that are imperfectly understood, such as upward propagating waves that break or other fluid dynamical instabilities (for example, magnetorotational instabilities, MRI, in the Moon-forming disk have been previously discussed, Charnoz and Michaut 2015; Carballido et al. 2016; Gammie et al. 2016). An upper bound in the convective region follows from consideration of heat flow (essentially the bound provided by convective vigor). For a characteristic fluid velocity $v_{\text {conv }}$ of $\left(F_{\text {conv }} / \rho\right)^{\frac{1}{3}} \sim$ $10^{2} \mathrm{~m} \mathrm{~s}^{-1}$, where $F_{\text {conv }}$ is the convective flux and $\rho$ is the density, and the scale height of the disk $\bar{H}$ of hundreds of km, we expect $K \sim v_{\text {conv }} \bar{H} \sim 10^{7} \mathrm{~m}^{2} \mathrm{~s}^{-1}$, but values in upper stably stratified regions can easily be many orders of magnitude lower. Simultaneously, the values can also be higher since the density is much lower (meaning that less energy per unit volume is required for mixing). The values of $K$ for planetary atmospheres are typically $10^{2}-10^{6} \mathrm{~m}^{2} \mathrm{~s}^{-1}$ Atreya, 1986; Moses et al., 2000; de Pater and Lissauer, 2010) but these are for much less energetic systems and for smaller characteristic length scales, and therefore a higher value is possible in our case. Fortunately, the value of $K$ does not matter much, as we now explain.

The upper limit of the escape flux for a light element $i$ (hydrogen), $\phi_{i}$ is approximately described as $\phi_{i} \leq \phi_{l} \sim b_{i j} f_{i} / H_{j}$ (Equation 1) as discussed in Section 1. Importantly, the parameters in this equation change rather little even as the pressure and number density at the homopause change by many orders of magnitude. It should be noted that Equation (1) has a simple physical interpretation. Except for factors of order unity, it indicates that the escape flux is bounded above by $\sim n c$, where $n$ is the number density of the atom or molecule in question and $c$ is the sound speed for that species, with the number density being evaluated at the place where the mean free path for the dominant species is of order $H$ (this is the so-called exobase).

We can approximately estimate the homopause location based on the following two scenarios. If the region of the disk is dominated by oxygen atoms and has a small fraction of hydrogen atoms at $2000 \mathrm{~K}$, then $D \sim K$ at $p \sim 10^{-3}-10^{1} \mathrm{~Pa}\left(m_{i}=1 \mathrm{~g} \mathrm{~mol}^{-1}, m_{j}=16 \mathrm{~g}\right.$ $\mathrm{mol}^{-1}, \sigma_{i}=2 \times 53 \mathrm{pm}$, and $\left.\sigma_{j}=2 \times 60 \mathrm{pm}\right)$. If the disk is dominated by $\mathrm{SiO}$ at $2000 \mathrm{~K}$, this condition is met at $p \sim 10^{-4}-10^{0} \mathrm{~Pa}\left(m_{i}=1 \mathrm{~g} \mathrm{~mol}^{-1}, m_{j}=30 \mathrm{~g} \mathrm{~mol}^{-1}, \sigma_{i}\right.$ $=2 \times 53 \mathrm{pm}$, and $\left.\sigma_{j}=320 \mathrm{pm}\right)$. Thus, the homopause pressure range is estimated as $\sim 10^{-4}-10^{1} \mathrm{~Pa}$ as discussed in Section 2.4

\section{A1.2. Further model validation}

There are further assumptions in the model in addition to the points raised in Section 4.3 . One of the underlying assumptions of hydrodynamic escape is that once the flow reaches its sound speed as it expands outwards and eventually escapes from the disk and Earth. According to previous studies (Walker, 1982; Zahnle et al., 1990), this approximation is likely to be valid if the exobase is above the critical point. The critical point is where the velocity reaches the sound velocity. This indicates that the criterion is $l_{c}<H_{1 c}$, where $l_{c}$ is the mean free path and $H_{1 c}$ is the scale height of a light element and the subscript $c$ describes the critical point. This is rewritten as (Zahnle et al., 1990)

$$
\phi_{l} \geq \frac{G M_{\oplus}}{r^{\prime 2}} \frac{m_{1}}{R T} \frac{4 b_{11}}{\sqrt{\pi}}
$$

This becomes $5.69 \times 10^{15}$ atoms $\mathrm{m}^{-2} \mathrm{~s}^{-1}\left(r^{\prime}=4.24 R_{\oplus}\right.$, $T=2000 \mathrm{~K}, m_{1}=1 \times 10^{-3} \mathrm{~g} \mathrm{~mol}^{-1}$ ), which is comparable to the hydrogen escape flux we estimate. It is possible that the escape flux becomes smaller than this value and thus the condition above may not be met. In this case, the hydrogen flux is very weak and heavy elements would not be dragged to space (Zahnle et al., 
1990). In other words, the diffusion-limited hydrogen escape rate is considered the upper limit and this makes our argument even stronger that hydrogen and volatile loss from the disk are minor.

Another assumption is that we ignore effects of dissociation on the lapse rate for simplicity (Section 2.3). The reaction of $\mathrm{SiO}_{2}=\mathrm{SiO}+\mathrm{O}$ is endothermic and the absolute value of the enthalpy is comparable to that of $\mathrm{SiO}_{2}$ condensation. This indicates that this reaction would affect the lapse rate, but this would still not change the final outcome because our result that the water escape is not efficient holds under the wide temperature range $(2500-4000 \mathrm{~K})$.

\section{References}

Abe, Y., Matsui, T., 1986. Early evolution of the Earth: Accretion, atmosphere formation, and thermal history. Journal of Geophysical Research 91, E291.

Atreya, S., 1986. Atmospheres and ionospheres of the outer planets and their satellites. Physics and Chemistry in Space .

Baraffe, I., Selsis, F., Chabrier, G., Barman, T.S., Allard, F., Hauschildt, P.H., Lammer, H., 2004. The effect of evaporation on the evolution of close-in giant planets. Astronomy and Astrophysics 419, L13-L16.

Bottke, W.F., Walker, R.J., Day, J.M.D., Desvorny, D., Elkins-Tanton, L., 2010. Stochastic late accretion to earth, the moon, and mars. Science 330, 1527-1530.

Boyce, J.W., Liu, Y., Rossman, G.R., Guan, Y., Eiler, J.M., Stolper, E.M., Taylor, L.A., 2010. Lunar apatite with terrestrial volatile abundances. Nature 466, 466-9.

Boyce, J.W., Tomlinson, S.M., McCubbin, F.M., Greenwood, J.P., Treiman, A.H., 2014. The lunar apatite paradox. Science 344, 400-2.

Cameron, A.G.W., Ward, W.R., 1976. The origin of the Moon. Lunar Planet. Sci. VII , 120.

Canup, R.M., 2012. Forming a Moon with an Earth-like composition via a giant impact. Science 338, 1052-1055.

Canup, R.M., Asphaug, E., 2001. Origin of the Moon in a giant impact near the end of the Earth's formation. Nature 412, 708-712.

Canup, R.M., Barr, A.C., Crawford, D.A., 2013. Lunar-forming impacts: High-resolution $\mathrm{SPH}$ and AMR-CTH simulations. Icarus 222, 200-219.

Canup, R.M., Visscher, C., Salmon, J., Fegley, B., 2015. Depletion of volatile elements in the moon due to incomplete accretion within an impact-generated disk. Nature Geoscience 8, 918-921.

Carballido, A., Desch, S.J., Taylor, G.J., 2016. Magneto-rotational instability in the protolunar disk. Icarus 268, 89-101.

Chamberlain, J., Hunten, D.M., 1987. Theory of Planetary Atmospheres, Second Edition. International Geophysics 36, 481.

Charnoz, S., Michaut, C., 2015. Evolution of the protolunar disk: Dynamics, cooling timescale and implantation of volatiles onto the earth. Icarus 260, 440-463.

Chase, M., Davies, C., Downey, J., Frurip, D., Mcdonald, R., Syverud, A., 1985. Janaf thermochemical tables 3rd edition. J. Phys. Chem. Ref. Data 14, 9271856.

Ćuk, M., Stewart, S.T., 2012. Making the Moon from a fast-spinning Earth: A giant impact followed by resonant despinning. Science 338, 1047-1052.

Dauphas, N., 2017. The isotopic nature of the Earth?s accreting material through time. Nature 541, 521-524.
Desch, S.J., Taylor, G.J., 2013. Isotopic mixing due to interaction between the protolunar disk and the Earth's atmosphere. Lunar Planet. Sci. Conf. 44th , 2566.

Elkins-Tanton, L.T., Grove, T.L., 2011. Water (hydrogen) in the lunar mantle: Results from petrology and magma ocean modeling. Earth and Planetary Science Letters 307, 173-179.

Elkins-Tanton, L.T., Linda, T., Burgess, S., Yin, Q.Z., 2011. The lunar magma ocean: Reconciling the solidification process with lunar petrology and geochronology. Earth and Planetary Science Letters 304, 326-336.

Fegley, B., Jacobson, N.S., Williams, K.B., Plane, J.M.C., Schaefer, L., Lodders, K., 2016. Solubility of rock in steam atmospheres of planets. The Astrophysical Journal 824, 103.

Gammie, C.F., Liao, W.T., Ricker, P.M., 2016. A hot big bang theory: magnetic fields and the early evolution of the protolunar disk. Astrophysical Journal 828, 58.

Genda, H., Abe, Y., 2003. Modification of a proto-lunar disk by hydrodynamic escape of silicate vapor. Earth Planets Space 55, 5357.

Genda, H., Ikoma, M., 2008. Origin of the ocean on the earth: Early evolution of water $\mathrm{d} / \mathrm{h}$ in a hydrogen-rich atmosphere. Icarus 194 , 42-52.

Hartmann, W.K., Davis, D.R., 1975. Satellite-sized planetesimals and lunar origin. Icarus 24, 504-514.

Hauri, E.H., Saal, A.E., Nakajima, M., Anand, M., Rutherford, M.J., van Orman, J.A., Le Voyer, M., 2017. Origin and evolution of water in the moon's interior. Annual Review of Earth and Planetary Sciences 45, 89-111.

Hauri, E.H., Saal, A.E., Rutherford, M.J., Van Orman, J.A., 2015. Water in the Moon's interior: Truth and consequences. Earth and Planetary Science Letters 409, 252-264.

Hauri, E.H., Weinreich, T., Saal, A.E., Rutherford, M.C., Van Orman, J.A., 2011. High pre-eruptive water contents preserved in lunar melt inclusions. Science 333, 213-5.

Herwartz, D., Pack, A., Friedrichs, B., Bischoff, A., 2014. Identification of the giant impactor Theia in lunar rocks. Science 344, $1146-50$.

Hui, H., Peslier, A.H., Zhang, Y., Neal, C.R., 2013. Water in lunar anorthosites and evidence for a wet early Moon. Nature Geosci. 6, 177-180.

Hunten, D.M., 1973. The Escape of Light Gases from Planetary Atmospheres. Journal of Atmospheric Sciences 30, 1481-1494.

Ito, Y., Ikoma, M., Kawahara, H., Nagahara, H., Kawashima, Y., Nakamoto, T., 2015. Theoretical emission spectra of atmospheres of hot rocky super-earths. The Astrophysical Journal 801, 144.

Karato, S.i., 2014. Asymmetric shock heating and the terrestrial magma ocean origin of the moon. Proceedings of the Japan Academy, Series B 90, 97-103.

Krähenbühl, U., Ganapathy, J., Morgan, J.W., Anders, E., 1973. Volatile Elements in Apollo 16 Samples: Possible Evidence for Outgassing of the Moon. Science 180, 858-861.

Kruijer, T.S., Kleine, T., Fischer-Godde, M., Sprung, P., 2015. Lunar tungsten isotopic evidence for the late veneer. Nature 520, 534-7.

Lin, Y., Tronche, E.J., Steenstra, E.S., van Westrenen, W., 2016. Evidence for an early wet Moon from experimental crystallization of the lunar magma ocean. Nature Geoscience 10, 14-18.

Lock, S.J., Stewart, S.T., Petaev, M.I., Leinhardt, Z.M., Mace, M., Jacobsen, S.B., Cuk, M., 2016. A new model for lunar origin: Equilibration with earth beyond the hot spin stability limit. Lunar and Planetary Science Conference 47th , 2881.

McCubbin, F.M., Steele, A., Hauri, E.H., Nekvasil, H., Yamashita, S., Hemley, R.J., 2010. Nominally hydrous magmatism on the Moon. Proc Natl Acad Sci U S A 107, 11223-8.

McDonough, W.F., Sun, S.S., 1995. The composition of the Earth. Chemical Geology 120, 223-253. 
Melosh, H.J., Sonnet, C.P., 1986. When worlds collide: Jetted vapor plumes and the moon's origin. Origin of the moon , 621-642.

Meyer, J., Elkins-Tanton, L., Wisdom, J., 2010. Coupled thermal?orbital evolution of the early moon. Icarus 208, 1-10.

Milliken, R.E., Li, S., 2017. Remote detection of widespread indigenous water in lunar pyroclastic deposits. Nature Geoscience 10, 561-565.

Moses, J., Bézard, B., Lellouch, E., 2000. Photochemistry of Saturn's Atmosphere I. Hydrocarbon Chemistry and Comparisons with ISO Observations. Icarus 143, 244-298.

Mukhopadhyay, S., 2012. Early differentiation and volatile accretion recorded in deep-mantle neon and xenon. Nature 486, 101-104.

Mundl, A., Touboul, M., Jackson, M.G., Day, J.M.D., Kurz, M.D., Lekic, V., Helz, R.T., Walker, R.J., 2017. Tungsten-182 heterogeneity in modern ocean island basalts. Science 356, 66-69.

Murray-Clay, R.A., Chiang, E.I., Murray, N., 2009. Atmospheric escape from hot jupiters. The Astrophysical Journal 693, 23-42.

Nakajima, M., Stevenson, D.J., 2014. Investigation of the Initial State of the Moon-Forming Disk: Bridging SPH Simulations and Hydrostatic Models. Icarus 233, 259-267.

Nakajima, M., Stevenson, D.J., 2015. Melting and mixing states of the Earth's mantle after the Moon-forming impact. Earth and Planetary Science Letters 427, 286-295.

Nakajima, S., Hayashi, Y., Abe, Y., 1992. A Study on the "Runaway Greenhouse Effect" with a One-Dimensional Radiative-Convective Equilibrium Model. Journal of Atmospheric Sciences 49, 22562266.

Pahlevan, K., Karato, S.i., Fegley, B., 2016. Speciation and dissolution of hydrogen in the proto-lunar disk. Earth and Planetary Science Letters 445, 104-113.

Pahlevan, K., Stevenson, D.J., 2007. Equilibration in the aftermath of the lunar-forming giant impact. Earth and Planetary Science Letters 262, 438-449.

Parker, E.N., 1963. Interplanetary Dynamical Processes. New York, Wiley, Chap. 4 , 41-50.

de Pater, I., Lissauer, J.J., 2010. Planetary Sciences. Cambridge University Press, 2 edition , 663.

Ringwood, A.E., Seifert, S., Wänke, H., 1987. A komatiite component in Apollo 16 highland breccias: implications for the nickelcobalt systematics and bulk composition of the Moon. Earth and Planetary Science Letters 81, 105-117.

Rizo, H., Walker, R.J., Carlson, R.W., Touboul, M., Horan, M.F., Puchtel, I.S., Boyet, M., Rosing, M.T., 2016. Early Earth differentiation investigated through $142 \mathrm{Nd}, 182 \mathrm{~W}$, and highly siderophile element abundances in samples from Isua, Greenland. Geochimica et Cosmochimica Acta 175, 319-336.

Robinson, K.L., Taylor, G.J., 2014. Heterogeneous distribution of water in the Moon. Nature Geoscience 7, 401-408.

Rufu, R., Aharonson, O., Perets, H.B., 2017. A multiple-impact origin for the moon. Nature Geoscience 10, 89-94.

Saal, A.E., Hauri, E.H., Van Orman, J.A., Rutherford, M.J., 2013. Hydrogen isotopes in lunar volcanic glasses and melt inclusions reveal a carbonaceous chondrite heritage. Science 340, 1317-20.

Sharp, Z.D., McCubbin, F.M., Shearer, C.K., 2013. A hydrogen-based oxidation mechanism relevant to planetary formation. Earth and Planetary Science Letters 380, 88-97.

Tera, F., Papanastassiou, D.A., Wasserburg, G.J., 1974. Isotopic evidence for a terminal lunar cataclysm. Earth and Planetary Science Letters 22, 1-21.

Thompson, C., Stevenson, D.J., 1988. Gravitational instability in twophase disks and the origin of the moon. Astrophys. J. 333, 452481

Touboul, M., Puchtel, I.S., Walker, R.J., 2012. 182W Evidence for Long-Term Preservation of Early Mantle Differentiation Products. Science 335, 1065-1069.
Touboul, M., Puchtel, I.S., Walker, R.J., 2015. Tungsten isotopic evidence for disproportional late accretion to the Earth and Moon. Nature 520, 530-3.

Visscher, C., Fegley, B., 2013. Chemistry of Impact-Generated Silicate Melt-Vapor Debris Disks. The Astrophysical Journal 767, L12.

Volkov, A.N., Johnson, R.E., Tucker, O.J., Erwin, J.T., 2011. Thermally driven atmospheric escape: Transition from hydrodynamic to jeans escape. The Astrophysical Journal 729, L24.

Walker, J.C.G., 1982. The earliest atmosphere of the Earth. Precarnbrian Research 17, 147-171.

Wang, K., Jacobsen, S.B., 2016. Potassium isotopic evidence for a high-energy giant impact origin of the moon. Nature 538, 487490.

Ward, W.R., 2012. On the vertical structure of the protolunar disk . Astron. J. 744, 140

Wiechert, U., Halliday, A.N., Lee, D.C., Snyder, G.A., Taylor, L.A., Rumble, D., 2001. Oxygen isotopes and the Moon-forming giant impact. Science 294, 345-348.

Wieczorek, M.A., Neumann, G.A., Nimmo, F., Kiefer, W.S., Taylor, G.J., Melosh, H.J., Phillips, R.J., Solomon, S.C., Andrews-Hanna, Jeffrey C. Asmar, S.W., Konopliv, A.S., Lemoine, F.G., Smith, D.E., Watkins, M.M., Williams, J.G., Zuber, M.T., 2013. The crust of the moon as seen by grail. Science 339, 671-675.

Willbold, M., Elliott, T., Moorbath, S., 2011. The tungsten isotopic composition of the Earths mantle before the terminal bombardment. Nature 477, 195-199.

Young, E.D., Kohl, I.E., Warren, P.H., Rubie, D.C., Jacobson, S.A., Morbidelli, A., 2016. Oxygen isotopic evidence for vigorous mixing during the Moon-forming giant impact. Science 351, 493-496.

Zahnle, K., Kasting, J.F., Pollack, J.B., 1990. Mass fractionation of noble gases in diffusion-limited hydrodynamic hydrogen escape. Icarus 84, 502-527. 